\title{
FUNDAMENTALS OF THE DEEP ROLLING OF COMPRESSOR BLADES FOR TURBO AIRCRAFT ENGINES
}

\author{
S. Mader, F. Klocke \\ 2005062 \\ Laboratory of Machine Tools and Production Engineering, WZL RWTH Aachen, \\ Chair of Manufacturing Technologies, Steinbachstraße 53, 52074 Aachen, Germany
}

\begin{abstract}
The weakening of the fatigue strength of turbine blades due to local impact damage caused by foreign objects (Foreign Object Damage - FOD) represents a significant safety risk in modern aviation. It is possible to employ deep rolling to counteract such component weakening in a particularly effective way.

In order to derive a possible process parameter-set in advance, fundamental knowledge regarding the elastic and plastic deformation was researched.

For this, FE analysis and practical tests were performed and compared. They are showing the complex deformation mechanisms occurring during the rolling contact.
\end{abstract}

\section{Subject Index}

deep-rolling, elastic and plastic deformation, fan and compressor blades, foreign object damage (FOD),

\section{Introduction}

The safety of turbo aircraft engines is influenced to a large extent by their tolerance to the damaging effects of foreign objects. Foreign object damage (FOD) occur above all within the fan, the first compressor stages and on the blade edges in particular. Notches, tensile residual stresses and sometimes even micro cracks are induced by FOD. Sometimes these damages lead to crack initiation and rapid crack growth upon subsequent application of alternating loads. This causes much earlier fracturing than is the case with undamaged blades [Martinez 2002, Bache 2002].

Strain hardening of the rim zone of the component is one promising way to suppress the crack formation resulting from alternating loads, as well as to stop or slow down crack growth [Altenberger 2003, Nalla 2003]. Furthermore, deep-rolled testpieces reveal the capacity to withstand almost double as many cycles of stress compared to samples strain-hardened by shot peening or laser shot peening [Nalla 2003, Shepard 2003]. This is largely attributable to the greater plastic strain that may be achieved.

Deep rolling of thin-walled fan and compressor blades is possible by using special rolling tool produced by the company ECOROLL (Fig. 1). This tool uses a hydrostatically supported ball as the rolling element. This is mounted in a suitable holder and supported by a hydraulic piston which, in turn, may be moved axially in a sleeve mounted on the tool shank and which ensures a constant rolling force corresponding to the hydraulic pressure.

In order to prevent the thin-walled blades from bending during the deep-rolling process, the rolling forces are compensated for by two opposed rolling tools.

The design of such deep-rolling processes is, however, associated with time- and cost-intensive test efforts as no suitable methods exist for deriving the necessary process variables from the desired rim zone properties. 


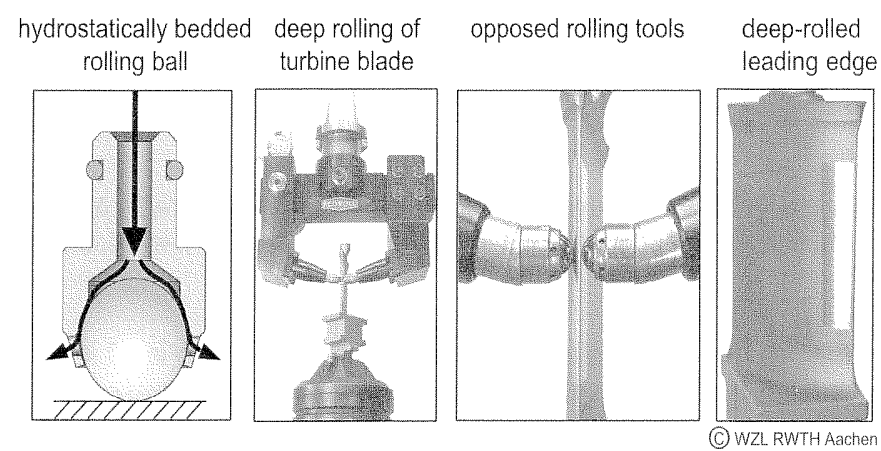

Fig. 1: Principle of a hydraulically driven and hydrostatically supported rolling tool and machining tests

One needed aspect is the knowledge regarding the elastic and plastic process taking place during the rolling contact. In spite of several research papers in which deeprolling in particular [Achmus 1999, Schaal 2002, Black 1997, Skalski 1995], or the contact in general between a sliding or rolling element and a workpiece that deforms plastically [Johnson 1985, loannides 1999] is examined, there is, to date, no fundamental understanding of the process.

In order to generate this fundamental knowledge and to be able to derive a possible process parameter-set in advance, the entire process was researched.

\section{Methods, Results and Discussion}

The experiments demonstrated that the deep-rolling process is largely based on a compression operation. The surface of the rolling ball is pressed orthogonally with the rolling force onto the surface. Should the workpiece have a rough surface, the first thing to occur is that micro compressions form between the ball and the roughness peaks. This leads to the generation of maximum equivalent stresses within the roughness peaks which soon assume high values and reach the yield point. This leads to a vertical plastic compression of the roughness peaks. At the same time, the roughness valleys in between are raised and their notches reduced.

3D-finite-element simulations show that, along with the levelling of the roughness peaks, a maximum equivalent stress is formed beneath the surface of the workpiece. As long as these stresses do not reach the yield point of the material, the stress field along the contact normals corresponds to the theory of elastic stresses from Hertz and Föppl.

As can be seen in Fig. 2, area "a", this maximum equivalent stress extends on the left and right of the contact normals (c.n.) towards the surface and ends directly next to the outer contact area (c.a.) between the ball and the workpiece.

If the rolling force is further increased, the equivalent stress reaches the yield point and plastic material deformation occurs beneath the ball, stretching laterally up to the surface of the workpiece.

A detailed analysis of the stress sites reveals a large area of vertical compressive stress directly beneath the rolling ball. This causes vertical plastic compression, leading to a depression in the surface of the workpiece, as well as to a horizontal 
extension of the material volume perpendicular to the direction of the compression (Fig. 2, area "b").

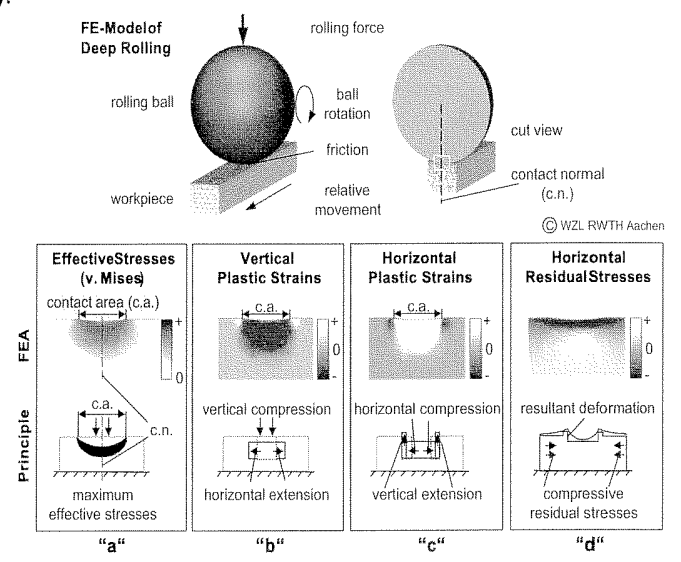

Fig. 2: FE analysis of deep rolling across the rolling line

The horizontal extension causes the material surrounding this area to be laterally displaced. This lateral displacement, in turn, causes the neighbouring area to undergo primarily elastic, horizontal compression. It is only in the area near to the surface, directly next to the contact zone between the ball and the workpiece, that small plastic horizontal compressions occur. This leads to vertical extension which spreads in a direction perpendicular to, i.e. vertically to, the deformation (Fig. 2, area "c") and results in an elevation of the areas surrounding the contact zone. The elevation is most pronounced in the area where plastic horizontal compression arises directly next to the contact zone.

The depression of the workpiece surface directly beneath the rolling ball that was observed in the FE simulation, together with the elevation of the area directly next to the contact zone of the ball, result in the formation of a crater geometry (Fig. 2, area "d"). This surface displacement matches observations from practical tests.

If the ball and workpiece now move relative to each other in a horizontal direction, the ball begins to roll on the surface. In so doing, the dominating effect of vertical compression remains, merely being superimposed on the workpiece surface by the previously horizontally compressed crater elevation that is then decreased in height and thereby vertically compressed. The results of the numerical process simulation may be seen in Fig. 3 in which a cross section of workpiece is shown, cut in the rolling direction.

As a result of local deformation of the workpiece and the associated change in the contact zone, the form of the maximum effective stress changes from an axially symmetric crescent shape to an asymmetrical droplet form, the largest area of which is located beneath the rolling ball and within the surface zone of the workpiece (Fig. 3, area "a"). This area stretches up to the surface of the workpiece and ends shortly in front of the contact zone of the rolling ball.

The dominating vertical compression occurs directly beneath the rolling ball (Fig. 3, area "b") and leads to permanent plastic horizontal extension in this area of the material (Fig. 3, area "c"). It is primarily in the area in front of the contact zone between the ball and the workpiece that this horizontal extension results in horizontal compression which reaches the yield point near to the surface. This causes the area near to the surface and in front of the contact area to undergo plastic horizontal 
compression. Shortly afterwards, the ball rolls over this area, thereby subjecting it to vertical compression (Fig. 3, area "b").

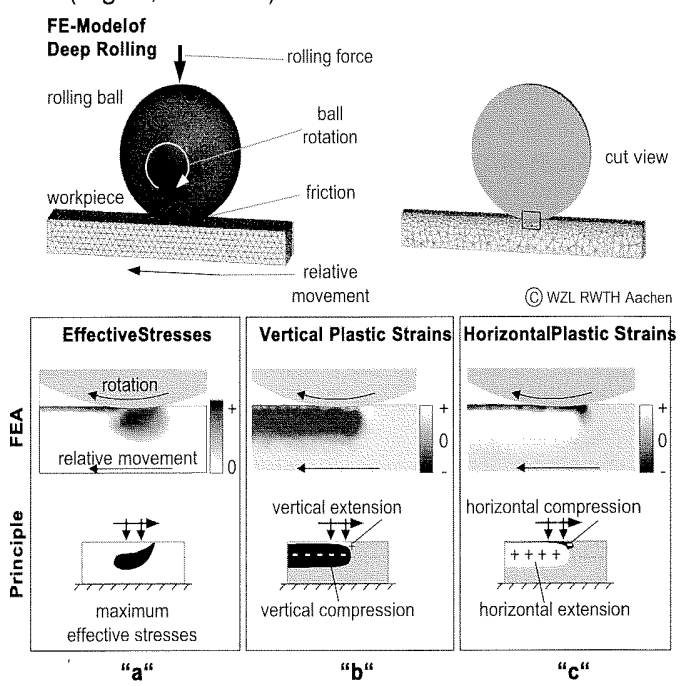

Fig. 3:FE analysis of deep rolling along the rolling line

Due to the two-fold deformation that occurs in opposing directions, this area stands out considerably from the rest of the deformed region, which only undergoes deformation in one direction.

The peculiarity of this area may be seen in micro hardness measurements, among other things. These were carried out in order to examine the crescent-shaped zone of plastic strain beneath a rolling line observed in the finite-element simulations. The plastic deformation at ambient temperature caused by the rolling process leads to strain hardening which plays a considerable role in the service life gains of dynamically loaded parts made from Ti6Al4V. This strain hardening effect is generated by the accumulation of dislocations. The effect may be seen, among other things, in an increase of local material hardness, which is why it may be revealed by means of hardness measurements.

Fig. 4 shows a field of micro hardness measurements carried out on such an alloy beneath a rolling track. An increase in the hardness can be seen clearly in the crescent-shaped area below the rolling ball which demonstrates a maximum hardness at its centre, beneath the surface. These measurement results verify the local plastic strain as calculated in the simulation (also shown) and thus underline the fact that the FE simulation represents the deformation processes in a qualitatively correct way.

Within the set of micro hardness measurements, a small area directly beneath the surface shows clearly discernible properties. It reveals a minimum hardness that lies below that of the basic material. Comparative measurements using both lower rolling forces, as well as different materials confirm this local decrease in hardness. It is clear, however, that two opposing compression processes which follow on from each other are what differentiate this area from the remaining area which is only subjected to one direction of compression. 
This could lead to material softening in this area, where first horizontal compression occurs in front of the ball, followed by vertical compression beneath the ball. Research of these deformation processes, which are decisive to the residual stresses near to the surface, will be undertaken in further studies.

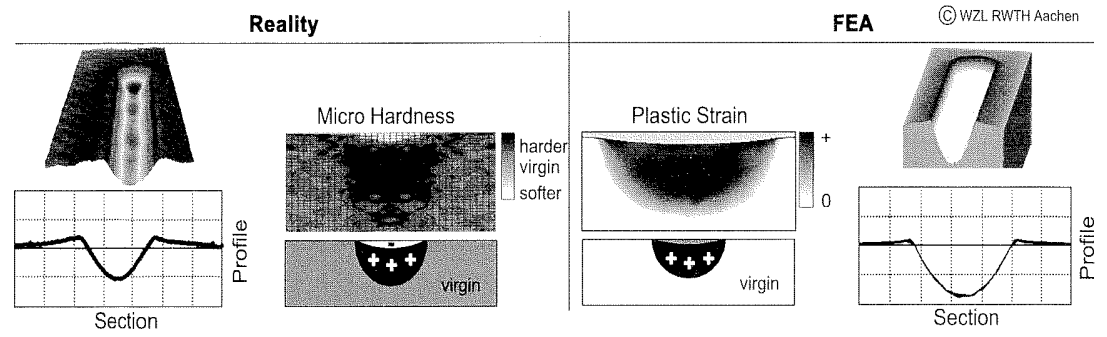

Fig. 4: Comparison of measured micro hardness and simulated plastic strain

Another significant effect of deep rolling is the generation of compressive residual stresses associated with local plastic deformation. This is caused by the extension of the vertically compressed material.

If several rolling lines are arranged next to each other, it is possible to deep roll a larger surface area. Studies have shown that burnished surfaces may be achieved by overlapping individual rolling tracks. On these surfaces, the single rolling tracks cannot be identified any more.

The strain hardening that occurs beneath such rolling fields is composed of individual rolling tracks overlapping each other, leading to higher overall levels of strain hardening. Furthermore, initial measurements on rolling fields with different degrees of overlap indicate that the residual stresses of individual rolling paths also appear to overlap each other. The increasing overlap of the rolling paths leads to a higher degree of permanent compressive residual stress in the surface zone of the component.

Many questions still remain unanswered, however, with respect to the influence of the individual rolling paths on each other. Of particular interest, for example, is the question as to what extent the rolling paths need to overlap each other in order to generate evenly strain-hardened layers and homogenous compressive residual stresses along the entire surface zone of the component.

This is of relevance because local zones with insufficient surface-zone properties would otherwise display a tendency towards crack initiation and crack growth.

High-resolution residual stress measurements will be carried out, among other things, in the near future in order to answer such questions.

In drawing up all of this fundamental data, it will be ultimately possible to develop a good qualitative understanding of the deep-rolling process. The finite element method will also assist in gaining some quantitative statements on deformation, strain hardening and residual stress.

All this will contribute to specifying process parameters more precisely.

However, as the quantitative correlation between these properties and the strainhardening behaviour that develops upon damage to a blade by a foreign object is only insufficiently known, a certain number of practical tests will remain unavoidable even in future.

More detailed practice tests and an optimised rolling tool will be presented during the conference. 


\section{Acknowledgements}

This research were supported by the German Research Foundation (Deutsche Forschungsgemeinschaft - DFG) within the framework of the Priority Programme 1146: "Modelling of Incremental Deformation Processes".

\section{References}

Martinez Martinez C. M., Eylon D., Nicholas T. et al. Effects of ballistic impact 20002 damage on fatigue crack initiation in Ti6Al4V; Materials Science and Engineering; A325; p. 465-477; 2002

Bache Bache M. R., Bradshaw C., Voice W., Characterisation of foreign 2002 object damage and fatigue strength in titanium based aerofoil alloys; Materials Science and Engineering; A354; p. 199-206; 2002

Altenberger Altenberger I., Stach E. A., Liu G., et al. An in situ transmission 2003 microscope study of the thermal stability of near-surface microstructures induced by deep rolling and laser-shock peening; Scripta Materialia; Nr. 48; p. 1593-1598; 2003

Nalla Nalla R. K., Altenberger I., Noster U. et al. On the influence of 2003 mechanical surface treatments -deep rolling and laser shock peeningon the fatigue behaviour of Ti6Al4V at ambient and elevated temperatures; Materials Science and Engineering; A 355; p. 216-230; 2003

Shepard Shepard M. J., Prevéy P. S., Jayaraman N., Effects of surface 2003 modifications on fretting fatigue properties of Ti6Al4V; $8^{\text {th }}$ International turbine engine HCF conference; 2003

Achmus Achmus C., Messung und Berechnung des Randschichtzustands 1999 komplexer Bauteile nach dem Festwalzen; Dissertation; TUBraunschweig; 1999

Schaal Schaal R., Berechnung der Dauerfestigkeit festgewalzter Bauteile 2002 durch FEM-Festwalzsimulation und Methoden der linearelastischen Bruchmechanik; Dissertation; TU-Darmstadt; 2002

Black Black A. J., Kopalinsky E. M., Oxley P. L. B., Analysis and 1997 experimental investigation of a simplified burnishing process; International Journal of Mechanical Sciences; Vol. 39; p. 629-635; 1997

Skalski Skalski K., Morawski A., Przybylski W., Analysis of contact elastic1995 plastic strains during the process of burnishing; International Journal of Mechanical Sciences; Vol. 37; p. 461-472; 1995

Johnson Johnson K. L., Contact mechanics; Cambridge University; 1985

1985

loannides loannides E., Bergling G., Gabelli A., An analytical formulation for the 1999 life of rolling bearings; Acta Polytechnica Scandinavica, Finnish Academy of Technology; Espoo; 1999 